\title{
INFLUENCIA DE LA COMPOSICIÓN QUÍMICA SUPERFICIAL DEL CARBÓN ACTIVADO EN LA ADSORCIÓN DE BENZOTIAZOLES
}

\section{INFLUENCE OF ACTIVATED CARBON CHEMICAL SURFACE COMPOSITION ON THE ADSORPTION OF BENZOTHIAZOLES}

\author{
Héctor Valdés ${ }^{1} \quad$ Claudio A. Zaror ${ }^{2}$ \\ Recibido 9 de diciembre de 2009, aceptado 18 de marzo de 2010 \\ Received: December 9, 2009 Accepted: March 18, 2010
}

\begin{abstract}
RESUMEN
Los benzotiazoles están esparcidos en el ambiente debido a su gran variedad de aplicaciones. Dentro de ellos, el benzotiazol (BT), el 2-hidroxibenzotiazol (OHBT), y el 2-metilbenzotiazol (MeBT) son conocidos como agentes tóxicos y poco biodegradables. La adsorción con carbón activado se plantea como una opción atractiva para la remoción de estos contaminantes. Sin embargo, existen dudas acerca del efecto de las propiedades químicas superficiales del carbón activado sobre la capacidad de adsorción de estos compuestos. Para el estudio se utilizó carbón activado granular Filtrasorb-400 modificado con el objetivo de obtener carbones con diferentes propiedades químicas superficiales. Las series de carbones activados fueron obtenidas por pretratamiento por desoxigenación y por oxidación con ozono a diferentes tiempos de contacto. El incremento en la concentración de los grupos oxigenados superficiales produce una disminución en la capacidad de adsorción de los tres contaminantes ensayados. Los resultados sugieren que el mecanismo de adsorción transcurre principalmente a través de las interacciones de dispersión $\pi-\pi$ entre los electrones del plano basal del carbón y el anillo aromático de los benzotiazoles. A valores de $\mathrm{pH}$ mayores al valor del $\mathrm{pH}$ de carga cero de la superficie del carbón activado $\left(\mathrm{pH}_{\mathrm{PZC}}\right)$, la capacidad de adsorción del carbón activado disminuye debido a la aparición de interacciones electrostáticas repulsivas entre los grupos superficiales oxigenados ionizados y las moléculas ionizadas de los benzotiazoles.
\end{abstract}

Palabras clave: Adsorción, benzotiazoles, carbón activado.

\begin{abstract}
Benzothiazoles, such as benzothiazole (BT), 2-hydroxybenzothiazole (OHBT), and 2-methylbenzothiazole (MeBT) are toxic and poorly biodegradable organic compounds, frequently present in wastewater from rubber related applications. Activated carbon adsorption has been identified as an attractive treatment to removing those contaminants. This paper presents experimental results on the effect of chemical surface properties of activated carbon on the adsorption mechanism of benzothiazoles. Activated carbons with different surface chemical composition were prepared from a Filtrasorb-400 activated carbon. Pre-treatment of activated carbon included de-oxygenation and ozonation with different ozone doses. Experimental results showed that high surface concentration of oxygenated electron withdrawing groups produced a significant reduction on carbon adsorption capacity of benzothiazoles. The results suggest that adsorption mechanism of benzothiazoles would mainly take place by $\pi-\pi$ dispersion interactions between the $\pi$-electrons of aromatic ring of benzothiazole molecules and the $\pi$-electrons of carbon graphene layers. When $p H$ was higher than point of zero charge $\left(\mathrm{pH}_{P Z C}\right)$ of the activated carbon, repulsive electrostatic interactions appeared between adsorbate and ionised oxygenated surface groups, lowering the carbon adsorption capacity of benzothiazoles.
\end{abstract}

Keywords: Activated carbon, adsorption, benzothiazoles.

\section{INTRODUCCIÓN}

Los benzotiazoles y sus derivados son compuestos orgánicos tóxicos y poco biodegradables. Debido a su gran variedad de aplicaciones los benzotiazoles están presentes en las aguas residuales de la industria de manufactura de aditivos de la goma [1], en las aguas residuales de las curtiembres y en los efluentes de las plantas de tratamiento

\footnotetext{
1 Facultad de Ingeniería. Universidad Católica de la Santísima Concepción. Alonso de Ribera 2850. Concepción, Chile. E-mail: hvaldes@ucsc.cl

2 Departamento de Ingeniería Química. Universidad de Concepción. Edmundo Larenas s/n. Concepción, Chile. E-mail: czaror@udec.cl
} 
[2-4]. Además, se pueden encontrar en los lixiviados de los residuos hospitalarios debido a la descomposición de los sellos de goma de las jeringas desechables y de los dispositivos de administración intravenosa [5]. Se ha reportado su presencia en suelos, sedimentos de estuarios y en aguas de ríos [6]. Desgraciadamente, los sistemas de tratamiento convencionales, basados en la actividad de los microorganismos, no son capaces de eliminar estos compuestos, ya que en su mayoría se ha demostrado que no son metabolizables como fuente de carbono y pueden ser adsorbidos por la membrana celular, derivando en su bioacumulación y biomagnificación [7]. Más aún, la presencia de estos compuestos puede inhibir la actividad de los microorganismos en dichos sistemas de tratamiento, reduciendo su capacidad depurativa [1,8]. Dado el carácter tóxico y recalcitrante de los benzotiazoles, es necesario utilizar tratamientos químicos de las aguas que lo contienen.

Dentro de las opciones de proceso para eliminar compuestos orgánicos tóxicos presentes en las aguas, se incluye el uso de carbón activado por su gran versatilidad debido a su alta área superficial, estructura porosa, alta capacidad de adsorción, y naturaleza química. Tradicionalmente se ha considerado a la porosidad y al área superficial los parámetros que definen la calidad del carbón activado. En la actualidad se ha demostrado que la química superficial del carbón activado juega un papel importante en el proceso de adsorción [9]. Una comprensión clara del mecanismo de adsorción es fundamental para el diseño y optimización de adsorbedores. Sin embargo, no existen datos disponibles acerca del efecto de los grupos superficiales del carbón activado en la adsorción de benzotiazoles, en vistas al desarrollo de adsorbentes más especializados y efectivos. Dentro de este contexto, el propósito del estudio que se presenta es investigar el efecto de la modificación de propiedades químicas superficiales del carbón activado en la capacidad de adsorción de los benzotiazoles. En particular, estudiar el efecto del $\mathrm{pH}$ sobre la capacidad de adsorción.

\section{METODOLOGÍA}

\section{Materiales}

Para el estudio se utilizó un carbón activado de origen bituminoso, Filtrasorb 400 (Calgon Carbon Corp., Pittsburgh, PA) con tamaño de partícula comprendida entre 500-800 $\mu \mathrm{m}$ (referido como muestra F"). El carbón fue lavado con agua desionizada, secado en estufa a $170{ }^{\circ} \mathrm{C}$ durante 24 horas, y almacenado en desecadora hasta su uso.

El ozono fue producido en un generador de ozono Ozocav $\left(5 \mathrm{~g} \mathrm{O}_{3} / \mathrm{h}\right)$. La determinación de ozono a la entrada y salida del reactor se realizó utilizando un espectrofotómetro
Spectronic modelo Genesys 5 con celda de flujo a una longitud de onda de $253,7 \mathrm{~nm}$.

BT (96\% de pureza), OHBT (99\% de pureza) y MeBT (99\% de pureza) fueron suministrados por Sigma-Aldrich. El ácido fosfórico y el hidróxido de sodio (ambos para análisis) fueron obtenidos de Merck. Todas las soluciones fueron preparadas con agua ultrapura obtenida de un sistema Millipore MilliQ.

\section{Pretratamiento de las muestras de carbón}

Con el objetivo de estudiar la influencia de los grupos oxigenados superficiales en el mecanismo de adsorción de los benzotiazoles sobre el carbón activo se prepararon carbones activados con diferentes composiciones químicas superficiales a partir de la muestra de carbón activado original Filtrasorb-400 (F). Se aplicaron básicamente dos pretratamientos al carbón original: oxidación por acción de ozono gaseoso y desoxigenación a $1.000{ }^{\circ} \mathrm{C}$ en atmósfera inerte.

El pretratamiento de oxigenación se llevó a cabo con ozono gaseoso en un reactor de lecho fijo. El reactor fue cargado con $2 \mathrm{~g}$ de carbón y operado bajo un flujo constante de ozono de $76 \mathrm{mg} \mathrm{O} / \mathrm{min}$ a $25^{\circ} \mathrm{C}$ y $1 \mathrm{~atm}$ de presión durante intervalos de 10,60, $120 \mathrm{~min}$ como ha sido descrito previamente [10]. Las muestras de carbón ozonizadas fueron identificadas como F-10, F-60, F-120. La desoxigenación de la superficie del carbón fue realizada en un horno tubular horizontal a $1.000{ }^{\circ} \mathrm{C}$ bajo una corriente de nitrógeno. Para ello, $13 \mathrm{~g}$ de muestra fueron colocados en un recipiente de cuarzo y llevados al horno. Los experimentos se realizaron a una velocidad de calentamiento de la muestra de $10{ }^{\circ} \mathrm{C} / \mathrm{min}$ bajo flujo constante de nitrógeno $\left(5 \mathrm{dm}^{3} / \mathrm{min}\right)$ y temperatura constante de $1.000{ }^{\circ} \mathrm{C}$ durante $60 \mathrm{~min}$. Posteriormente, la muestra fue enfriada a temperatura ambiente en atmósfera de nitrógeno y almacenada en desecadora bajo vacío hasta su uso. Esta muestra de carbón se denominó F-G. En la Tabla 1 se presenta un resumen de los códigos utilizados para la identificación de las muestras de carbón utilizadas.

Tabla 1. Identificación de las muestras de carbón utilizadas

\begin{tabular}{|l|l|}
\hline Código & \multicolumn{1}{|c|}{ Muestras } \\
\hline F & Carbón activado original Filtrasorb-400 \\
\hline F-10 & Filtrasorb-400 ozonizado por $10 \mathrm{~min}$. \\
\hline F-60 & Filtrasorb-400 ozonizado por $60 \mathrm{~min}$. \\
\hline F-120 & Filtrasorb-400 ozonizado por $120 \mathrm{~min}$. \\
\hline F-G & Filtrasorb-400 desoxigenado \\
\hline
\end{tabular}




\section{Caracterización de las muestras de carbón}

Las propiedades texturales de los carbones activados fueron obtenidas por adsorción de $\mathrm{N}_{2}$ a $77 \mathrm{~K}$ utilizando un aparato de adsorción Micromeritics modelo Gemeni 2370. Se utilizaron muestras de aproximadamente $0,2 \mathrm{~g}$, previamente desgasificadas a $350{ }^{\circ} \mathrm{C}$ por 3 horas bajo flujo de nitrógeno. Los datos de área superficial aparente $\left(A_{B E T}\right)$ de las muestras de carbón fueron calculados a partir de los datos de adsorción de nitrógeno utilizando la ecuación de BET. Los volúmenes de microporos $\left(V_{\text {micro }}\right)$ y mesoporos $\left(V_{\text {meso }}\right)$ y el área de superficial correspondiente a los mesoporos $\left(\alpha_{\mathrm{s}}\right)$ fueron determinados según el método- $\alpha$ [11]. Las propiedades químicas superficiales fueron determinadas por neutralización ácido/base, espectroscopía de fotoelectrones de rayos-X (XPS) $\left(4 \cdot 10^{-9}\right.$ mbar, paso de energía 25-50 eV), y el pH del punto cero de carga $\left(\mathrm{pH}_{\mathrm{PZC}}\right)$, siguiendo los procedimientos descritos en una publicación anterior [10].

\section{Determinación de las isotermas de adsorción}

Las isotermas de equilibrio de adsorción en solución acuosa para el BT, OHBT, MeBT sobre las muestras de carbón activado fueron determinadas a $25 \pm 1{ }^{\circ} \mathrm{C}$ utilizando el método del punto en el recipiente [12]. Cantidades diferentes de carbón fueron pesadas y adicionadas en ocho frascos Erlenmeyer de $250 \mathrm{~cm}^{3}$ y $50 \mathrm{~cm}^{3}$ de solución acuosa del compuesto orgánico (30 $\mathrm{mg} / \mathrm{L}$ ) fueron añadidas a cada frasco. Las isotermas de adsorción fueron realizadas en agua ultrapura sin ajuste de $\mathrm{pH}$. En cada experimento se incluyeron dos frascos con la solución de los contaminantes orgánicos sin carbón activado con el objetivo de evaluar la posible volatilización o adsorción del adsorbato en las paredes del recipiente. Las suspensiones fueron agitadas hasta que se alcanzó el equilibrio (aproximadamente 48 horas, según resultados obtenidos en experimentos cinéticos preliminares). Finalmente, la concentración residual de adsorbato fue medida por espectrofotometría UV a una longitud de onda de $230 \mathrm{~nm}$.

\section{RESULTADOS Y DISCUSIÓN}

Caraterización de las muestras de carbón

En la Tabla 2 se resumen las características físicas y químicas de las muestras de carbón activado utilizadas. El pretratamiento con ozono disminuyó el volumen de microporos y el área BET. Por otro lado, el volumen de mesoporos y el área externa mostraron un ligero incremento debido a la ozonización, aunque el crecimiento no es proporcional al tiempo de la ozonización. La reacción de oxidación con ozono de la superficie del carbón activado parece ser la responsable de esos efectos.

La ozonización prolongada de la superficie de los carbones activados lleva a la formación de especies volálites [10]. Sin embargo, el tratamiento de desoxigenación de la muestra original llevó a un desarrollo de la porosidad del carbón con el correspondiente incremento en su área superficial. El volumen de microporos y mesoporos se incrementó en un $7 \%$ y $74 \%$, respectivamente, llevando a un 9,8\% de incremento en el área BET respecto al carbón activado original. Los cambios en la porosidad se deben a una gasificación parcial de la muestra [10]. Durante el tratamiento térmico los complejos superficiales oxigenados se descomponen en $\mathrm{CO}, \mathrm{CO}_{2}$ y $\mathrm{H}_{2} \mathrm{O}[10]$.

Las propiedades ácidas y básicas de los carbones activados se muestran en la Tabla 2. Se aprecia un aumento en el contenido de grupos ácidos producto del pretratamiento por ozonización, mientras que la concentración de grupos básicos se reduce significativamente. En general, el comportamiento ácido ha sido asociado con estructuras tales como carboxilos, carbonilos, fenoles, lactonas y anhídridos de ácidos carboxílicos [13]. Por otra parte, el comportamiento básico se ha relacionado con otras funciones que contienen oxígeno, tales como estructuras del tipo cromeno y $\gamma$-pirona [14] y con la presencia de electrones $\pi$ deslocalizados sobre el plano basal de la superficie del carbón activado [15].

Tabla 2. Características de las muestras de carbón activado utilizadas.

\begin{tabular}{|c|c|c|c|c|c|c|c|}
\hline Muestras & $\begin{array}{c}\mathbf{A}_{\text {BET' }} \\
\mathbf{m}^{2} / g\end{array}$ & $\begin{array}{c}\alpha_{\mathbf{s}}, \\
\mathbf{m}^{2} / g\end{array}$ & $\begin{array}{l}V_{\text {micro }}, \\
\mathrm{cm}^{3} / \mathrm{g}\end{array}$ & $\begin{array}{l}V_{\text {meso }} \\
\mathbf{c m}^{3} / g\end{array}$ & $\begin{array}{c}\text { Sitios ácidos, } \\
\mu \mathrm{eq} / \mathrm{g}\end{array}$ & $\begin{array}{c}\text { Sitios básicos, } \\
\mu \mathrm{eq} / \mathrm{g}\end{array}$ & $\mathbf{p H}_{\mathrm{PZC}}$ \\
\hline $\mathrm{F}$ & 1000 & 54 & 0,474 & 0,019 & 234 & 570 & 8,82 \\
\hline F-10 & 1023 & 87 & 0,472 & 0,031 & 702 & 437 & 5,96 \\
\hline F-60 & 815 & 72 & 0,380 & 0,025 & 2059 & 171 & 3,89 \\
\hline F-120 & 632 & 64 & 0,297 & 0,023 & 3370 & - & 2,59 \\
\hline F-G & 1098 & 94 & 0,505 & 0,033 & 0 & 801 & 10,36 \\
\hline
\end{tabular}


La exposición del carbón activado al ozono modifica la distribución de los grupos superficiales del carbón. El contenido de grupos fenólicos disminuye con el tiempo de ozonización, generándose grupos más oxidados como lactonas, grupos carbonilos y carboxílicos. Una información más detallada del efecto de la ozonización en las propiedades superficiales del carbón " $F$ " fue obtenida utilizando XPS y está presentada en otra publicación [10]. La disminución en el pH del punto cero de carga en los carbones oxidados está relacionada con el incremento en el número de sitios ácidos en la superficie del carbón.

Sin embargo, el procedimiento de desoxigenación provoca la eliminación de todos los complejos oxigenados ácidos de la muestra de carbón original que pueden ser detectados por el método de neutralización ácido/base, mientras que el carácter básico aumentó.

Este incremento se puede atribuir, primero, a la eliminación de los grupos ácidos. Otro factor puede ser la formación de óxidos superficiales básicos con estructuras tipo cromeno o $\gamma$-pirona por descomposición de los sitios oxigenados ácidos [16]. Además, a las altas temperaturas de gasificación la eliminación de los complejos oxigenados deslocaliza los electrones del plano basal, formándose sitios superficiales con una gran habilidad para donar pares de electrones y comportarse como base de Lewis [15]. El pretratamiento de desoxigenación elevó el $\mathrm{pH}_{\mathrm{PZC}}$ debido al incremento en los sitios básicos.

\section{Análisis de la adsorción}

En la Figura 1 se presentan las isotermas de adsorción sobre el carbón activado original (F) del BT, del OHBT y del MeBT a pH 5. Se aprecia que la máxima adsorción tiene lugar para el OHBT seguido por el MeBT, siendo el menos adsorbido el BT. El incremento en la capacidad de adsorción del carbón activado está vinculado con la presencia de los sustituyentes metilo o hidroxilo en la molécula de benzotiazol.

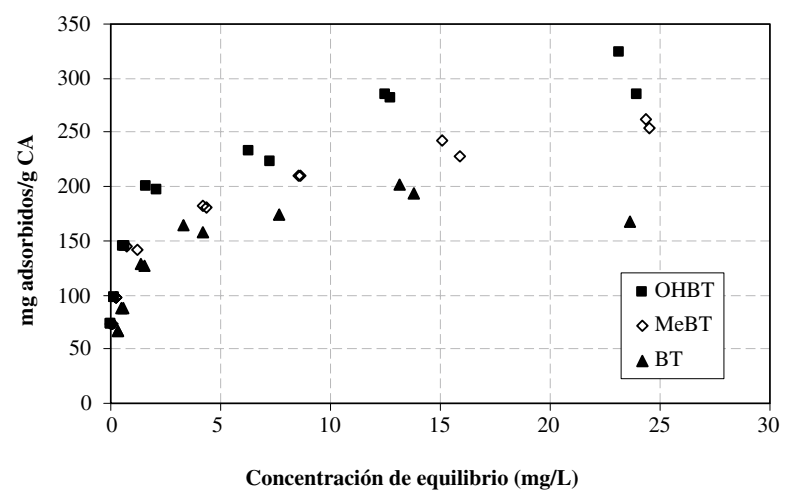

Figura 1. Isotermas de adsorción de benzotiazoles sobre el carbón activado original.
El carbón activado Filtrasorb 400 presenta un carácter básico $\mathrm{pH}_{\text {PZC }}$ cercano a 9. El incremento en la capacidad de adsorción del OHBT con respecto al MeBT y al BT, se pudiera relacionar con el aumento en la densidad electrónica en el sistema aromático. El grupo hidroxilo aumenta en mayor grado la densidad electrónica del sistema aromático en comparación al grupo metilo. La presencia de los grupos hidroxilo y metilo en la molécula de benzotiazol provocan un aumento en la densidad electrónica del sistema aromático de la molécula de BT, incrementando la capacidad de adsorción del carbón activado original. El incremento en la capacidad de adsorción estaría vinculado con el fortalecimiento de las fuerzas de dispersión entre el sistema de electrones $\pi$ del anillo aromático del benzotiazol y la banda $\pi$ de electrones de los planos grafíticos del carbón. Estos resultados
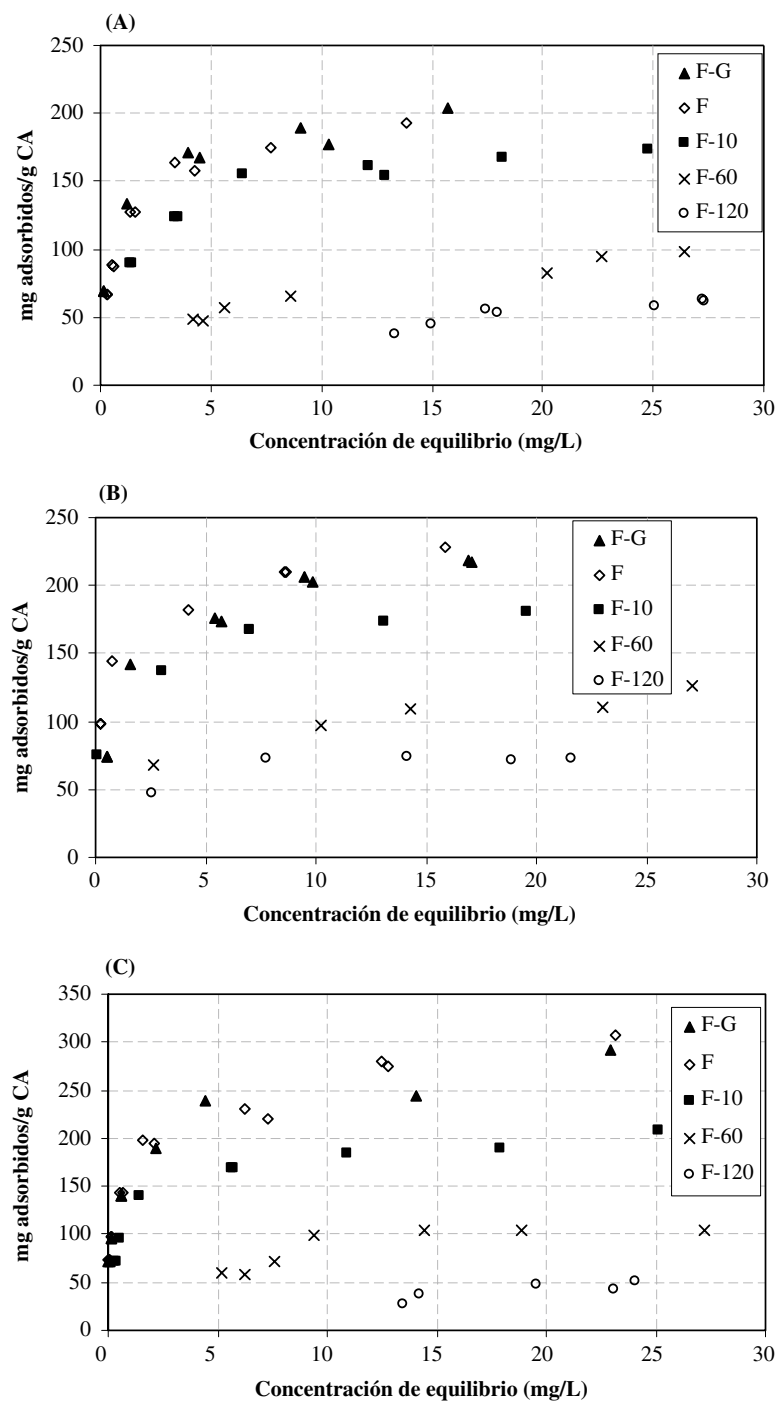

Figura 2. Isotermas de adsorción de benzotiazoles sobre carbones activados. (A) BT, (B) MeBT, (C) OHBT. 
concuerdan con el mecanismo de adsorción propuesto inicialmente por Coughlin y Ezra (1968) [17].

Las Figuras 2A, 2B y 2C muestran las isotermas de adsorción del BT, MeBT y OHBT, respectivamente, sobre carbones activados sometidos a pretratamiento con ozono por $10 \mathrm{~min}$ (F-10), 60 min (F-60), 120 min (F-120) y desoxigenado a $1.000{ }^{\circ} \mathrm{C}$ bajo flujo de nitrógeno (F-G).

Con un tiempo prolongado de exposición al ozono las funciones más oxidadas, como carbonilos y carboxilos, aumentan su concentración sobre la superficie del carbón activado. Estos grupos son electrón-atrayentes y provocan una disminución en la densidad electrónica del plano basal del carbón así como una disminución del $\mathrm{pH}_{\mathrm{PZC}}$.

La exposición prolongada del carbón activado al ozono gaseoso produce una disminución en la capacidad de adsorción de los tres contaminantes ensayados. La oxidación de los grupos funcionales superficiales del carbón provoca el debilitamiento en las interacciones de dispersión $\pi$ - $\pi$ entre el plano basal del carbón y el anillo aromático de los benzotiazoles debido a la disminución en la densidad electrónica de la superficie.

\section{Efecto del pH}

El efecto del pH sobre la capacidad de adsorción del BT, MeBT y OHBT sobre la serie de carbones activados estudiados se presenta en las Figuras 3A, 3B y 3C, respectivamente. Las flechas mostradas en las figuras señalan el valor del $\mathrm{pH}_{\mathrm{PZC}}$ de cada una de las muestras de carbón.

El aumento en la concentración de funciones oxidadas lleva a la disminución del $\mathrm{pH}_{\mathrm{PZC}}$ del carbón (ver Tabla 2) y en dependencia del $\mathrm{pH}$ de la solución a la ionización de estos grupos que incrementarían las interacciones electrostáticas repulsivas en un medio acuoso.

La adsorción de BT y del MeBT sobre el carbón activado desoxigenado y original no se ve mayormente afectado por el pH de la solución. La concentración de grupos protonizables en la superficie del carbón activado y la carga sobre la molécula de BT es baja, lo que se traduce en interacciones electrostáticas repulsivas débiles. Sin embargo, los carbones activados sometidos a altas dosis de ozono muestran una disminución en la capacidad de adsorción tanto del BT como del MeBT en la medida que se incrementa el pH. El incremento de grupos ionizables en la superficie de los carbones activados provoca la aparición de las interacciones electrostáticas repulsivas con las moléculas de los benzotiazoles, disminuyendo la adsorción.
En la Figura 3C se analiza el efecto del pH en la adsorción del OHBT. Se aprecia que la adsorción bajo condiciones $\mathrm{pH}<\mathrm{pH}_{\mathrm{PZC}}$ es mayor que cuando se realiza a $\mathrm{pH}>\mathrm{pH}_{\mathrm{PZC}}$. Para valores de $\mathrm{pH}>\mathrm{pH}_{\mathrm{PZC}}$ la superficie del carbón se carga negativa debido a la ionización de los grupos funcionales oxigenados. Por otra parte, el OHBT se ioniza en función del $\mathrm{pH}$ de la solución, $\mathrm{p} K_{a}=$ 8,65 [18]. A pH > 9 el OHBT se encuentra ionizado, disminuyendo la adsorción debido a la aparición de interacciones electrostáticas repulsivas.
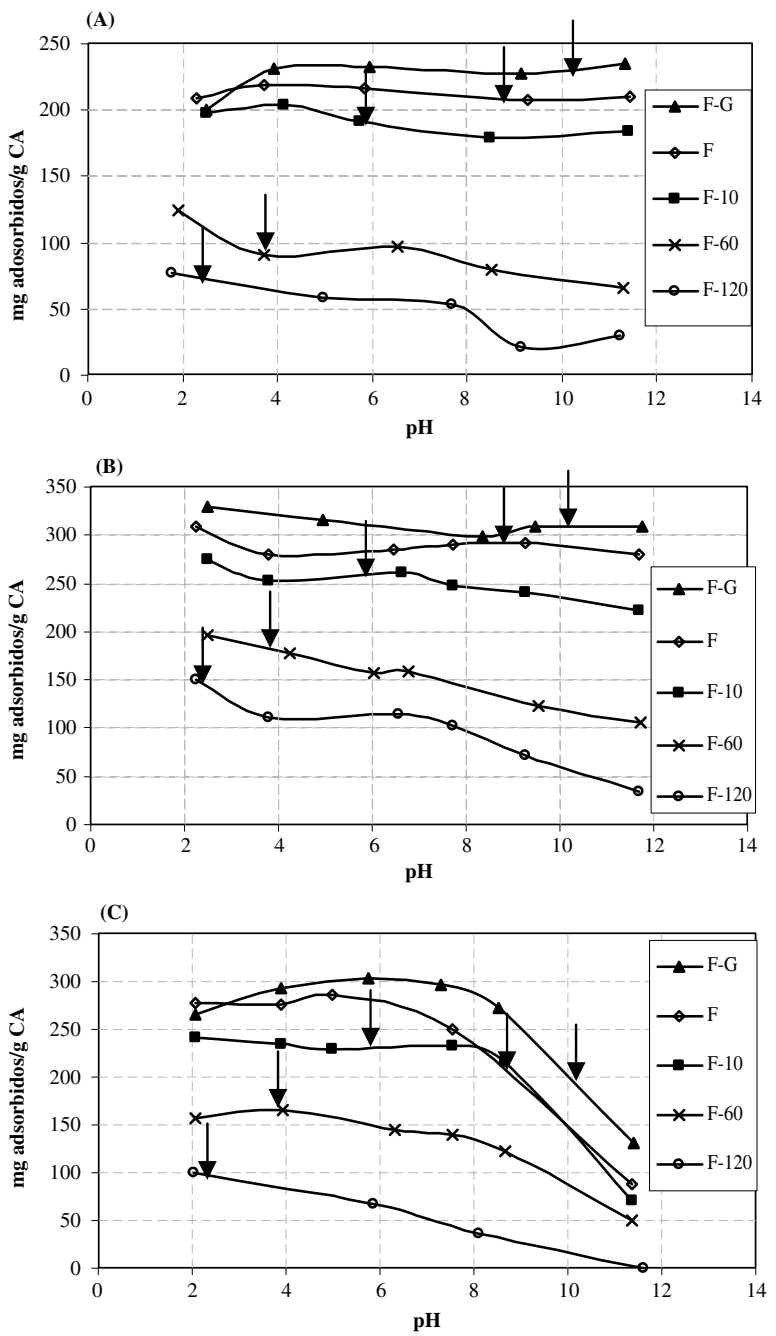

Figura 3. Influencia del pH en la capacidad de adsorción de benzotiazoles sobre carbones activados (A) BT, (B) MeBT, (C) OHBT.

\section{CONCLUSIONES}

El análisis de la adsorción de los benzotiazoles mostró que la adsorción de benzotiazoles fue afectada por la modificación de los grupos superficiales del carbón. En los carbones activados oxidados con ozono se reduce la 
capacidad de adsorción, lo que se atribuye a la disminución de las interacciones de dispersión debido a la disminución de la densidad de electrones $\pi$ en el plano basal del carbón. El tratamiento de desoxigenación de la superficie del carbón elimina los grupos funcionales oxigenados electrón-atrayentes incrementando las interacciones de dispersión por el aumento de la densidad de electrones $\pi$. Se demuestra que el mecanismo de adsorción de los benzotiazoles bajo condiciones de $\mathrm{pH}<\mathrm{pH}_{\mathrm{PZC}}$ transcurre fundamentalmente mediante interacciones $\pi-\pi$ entre la nube electrónica del benzotiazol y los electrones del plano basal del carbón a diferencia cuando el $\mathrm{pH}>\mathrm{pH}_{\mathrm{PZC}}$ las interacciones electrostáticas serían las responsables principales de la disminución de la adsorción de los benzotiazoles sobre el carbón activado.

\section{AGRADECIMIENTOS}

Los autores agradecen a FONDECYT (Proyectos $\mathrm{N}^{\mathrm{os}} 1060304$ y 1060892) por el apoyo brindado en el desarrollo de la investigación.

\section{REFERENCIAS}

[1] H. de Wever and H. Verachtert. "Biodegradation and toxicity of benzothiazoles". Wat. Res. Vol. 31, pp. 2673-2684. 1997.

[2] A. Kloepfer, M. Jekel and T. Reemtsma. "Determination of benzothiazoles from complex aqueous samples by liquid chromatography-mass spectrometry following solid-phase extraction". J. Chromatogr. A. Vol. 1058, pp. 81-88. 2004.

[3] A. Kloepfer, R. Gnirss, M. Jekel and T. Reemtsma. "Occurrence of benzothiazoles in municipal wastewater and their fate in biological treatment". Water Sci. Technol. Vol. 50, pp. 203-208. 2004.

[4] A. Kloepfer, M. Jekel and T. Reemtsma. "Occurrence, sources, and fate of benzothiazoles in municipal wastewater treatment plant". Environ. Sci. Technol. Vol. 39, pp. 3792-3798. 2005.

[5] C.B. Airaudo, A. Gayté-Sorbier and R. Momburg P. "Leaching of antioxidants and vulcanization accelerators from rubber closures into drug preparations". J. Biomat. Sci. Polym. Vol. 1, pp. 231-241. 1990.

[6] T. Reemtsma and M. Jekel. "Dissolved organics in tannery wastewater and their alteration by a combined anaerobic and aerobic treatment". Wat. Res. Vol. 31, pp. 1035-1046. 1997.
[7] M.A. Gaja and J.S. Knapp. "Removal of 2-Mercaptobenzothiazole by activated sludge: a cautionary note". Wat. Res. Vol. 32, Issue 12, pp. 3786-3789. 1998.

[8] H. De Wever, S. Weiss, T. Reemtsma, J. Vereecken, J. Müller, T. Knepper, O. Rörden, S. Gonzalez, D. Barcelo and M.D. Hernando. "Comparison of sulfonated and other micropollutants removal in membrane bioreactor and conventional wastewater treatment”. Water Res. Vol. 41, pp. 935-945. 2007.

[9] L.R. Radovic, C. Moreno-Castilla and J. RiveraUtrilla. "Carbon materials as adsorbents in aqueous solutions". Chem. Phys. Carbon. Vol. 27, pp. 227405. 2000.

[10] H. Valdés, M. Sánchez-Polo, J. Rivera-Utrilla and C.A. Zaror. "Effect of ozone treatment on surface properties of activated carbon". Langmuir. Vol. 18, pp. 2111-2116. 2002.

[11] F. Rodríguez-Reinoso, J.M. Martín-Martínez, C. Prado-Burguete and B. McEnaney. "A standard adsorption isotherm for the characterization of activated carbons". J. Phys Chem. Vol. 91, pp. 515516. 1987.

[12] C.-C. Leng and N.G. Pinto. "Effects of surface properties of activated carbons on adsorption behavior of selected aromatic". Carbon. Vol. 35, pp. 1375-1385. 1997.

[13] S.S. Barton, M. J. B. Evans, E. Halliop and J.A.F. MacDonald. "Acidic and basic sites on the surface of porous carbon”. Carbon. Vol. 35, pp. 1361-1366. 1997.

[14] H.P. Boehm. "Surface oxides on carbon and their analysis: a critical assessment". Carbon. Vol. 40, pp. 145-149. 2002.

[15] C.A. León y León, J.M. Solar, V. Calemma and L.R. Radovic. "Evidence for the protonation of basal plane sites on carbon". Carbon. Vol. 30, pp. 797-811. 1992.

[16] E. Papirer, S. Li and J.B. Donnet. "Contribution to the study of basic surface groups on carbons". Carbon. Vol. 25, pp. 243-247. 1987.

[17] R.W. Couglhin and F.S. Ezra. "Role of surface acidity in the adsorption of organic pollutants on the surface of carbon". Environ. Sci. Tecnol. Vol. 2, pp. 291-298. 1968.

[18] R. Andriozzi, V. Caprio and R. Marotta. "Oxidation of benzothiazole, 2-mercaptobenzothiazole and 2-hydroxibenzothiazole in aqueous solution by means of $\mathrm{H}_{2} \mathrm{O}_{2} / \mathrm{UV}$ or photoassisted Fenton systems". J. Chem. Technol. Biotechnol. Vol. 76, pp. 196-202. 2001. 